doi.org/ 10.51891/rease.v7iro.2845

\title{
A IMPORTÂNCIA DA EDUCAÇÃO FÍSICA NA VIDA DE CRIANÇAS OBESAS
}

\author{
Willian Vieira da Silva ${ }^{1}$ \\ Leonardo Squinello Nogueira Veneziano ${ }^{2}$
}

RESUMO: Nos últimos 30 anos, as prevalências de sobrepeso e obesidade cresceram de maneira assustadora no público infantil, e um dos principais motivos por tal aumento é o sedentarismo. Os alarmantes índices de obesidade infantil registrados no Brasil demonstram como é importante a prevenção do sobrepeso, o que torna a escola o local considerado ideal para realizar as intervenções necessárias, principalmente através da Educação Física, pois boa parte das crianças obesas ou com tendência, frequentemente tendem a se esconder e não participam das atividades do dia a dia. De forma que o professor das atividades físicas pode ser o impulsionador desses alunos, incentivando-os a uma prática física saudável. Portanto, o presente estudo tem como objetivo analisar a importância da educação física escolar e suas contribuições no controle da obesidade infantil, visto que, as aulas de Educação Física pela sua ampla aceitação tende a se tornar um dos poucos lugares possíveis para a prática de medidas de combate a obesidade infantil. Para alcançar esse objetivo, utilizou-se a pesquisa documental, analisando referenciais já existentes que contribuem para a discussão sobre o tema aqui analisado. Ao final, pode-se concluir que a Educação Física pode exercer se apresenta como meio bastante eficaz na ação de combate a obesidade infantil, desde que planejada, trazendo-lhes o conhecimento sobre os benefícios preventivos da atividade física em relação ao corpo saudável e utilizando métodos atuais, onde o prazer da atividade física possa ser o grande expoente de toda aula ministrada.

Palavras-chave: Obesidade. Educação Física. Hábitos Saudáveis. Atividade Física.

ABSTRACT: In the last 30 years, the prevalence of overweight and obesity has grown alarmingly in children, and one of the main reasons for this increase is sedentary lifestyles. The alarming rates of childhood obesity recorded in Brazil show how important it is to prevent overweight, which makes the school the place considered ideal to perform the necessary interventions, especially through Physical Education, because a good number of obese children or those with tendencies often tend to hide and not participate in daily activities. So that the teacher of physical activities can be the booster of these students, encouraging them to a healthy physical practice. Therefore, this study aims to analyze the importance of school physical education and its contributions to the control of childhood obesity, since physical education classes, due to their wide acceptance, tend to

\footnotetext{
Acadêmico do Curso de Fisioterapia da UniBRÁS Faculdade de Rio Verde. E-mail: wilhyansilva@hotmail.com

${ }^{2}$ Professor Mestre do Curso de Educação Física da UniBRÁS Faculdade de Rio Verde e orientador da pesquisa.
} 
become one of the few possible places for the practice of measures to combat childhood obesity. To achieve this goal, documentary research was used, analyzing existing references that contribute to the discussion on the subject analyzed here. In the end, it can be concluded that Physical Education can be a very effective way to combat childhood obesity, provided that it is planned, bringing them knowledge about the preventive benefits of physical activity in relation to a healthy body and using current methods, where the pleasure of physical activity can be the great exponent of every class taught.

Keywords: Obesity. Physical Education. Healthy Habits. Physical Activity.

\section{INTRODUÇÃO}

Mudanças socioeconômicas, geográficas, políticas e tecnológicas ocorridas nos últimos anos resultaram em relevantes transformações nos hábitos de saúde da população brasileira como atividade física e alimentação. Em consequência desse processo, o consumo de alimentos calóricos, com alto teor de açúcares, gorduras e sal, pobres em nutrientes tornou-se padrão comum de alimentação entre as famílias. E com isso, a obesidade infantil tem aumentado cada vez mais, tornando-se uma preocupação mundial (BRASIL, 2012).

Nos últimos 30 anos, as prevalências de sobrepeso e obesidade cresceram de forma assustadora. O modo de viver da sociedade moderna tem determinado um padrão alimentar que, associado ao sedentarismo, não é favorável à saúde da população. Estima-se que $20 \%$ das crianças brasileiras sejam obesas e cerca de $30 \%$ da população adulta apresentem algum grau de excesso de peso, sendo $25 \%$ dos casos mais graves (BRASIL, 2012).

Conceitualmente, a obesidade pode ser considerada como acúmulo de tecido gorduroso, regionalizado ou em todo corpo. Contudo, para uma criança é mais do que isso. A obesidade para esse grupo de pessoas pode acarretar em distúrbios psicológicos, pode afetar seu desenvolvimento motor, pode comprometer sua autoestima, implicar em problemas de saúde, entre outros fatores (CORNACHIONI; ZADRA; VALENTIM, 20II).

A propensão genética, hábitos alimentares inadequados, o sedentarismo, o estresse, o etilismo, entre outros fatores são apontados como os responsáveis pelo excesso de tecido adiposo no corpo, o qual tem a capacidade de gerar graves à saúde do indivíduo (MALTA, 20II). 
Segundo a Organização Mundial da Saúde (OMS) (2010), a obesidade tornou-se atualmente uma epidemia que se espalha em todo o mundo, sendo foco de inúmeros estudos que abordam sua prevenção e tratamento, tais como dietas, medicamentos e prática de atividade física.

Como o sobrepeso infantil está intimamente ligado a fatores externos, é de extrema importância o papel de um profissional de educação física que auxilie tanto no processo de emagrecimento, quanto na orientação da atividade física tirando a criança de frente de aparelhos eletrônicos e promovendo nela à vontade de praticar atividade física (ATALLA, 2016).

Nesse contexto, a escolha desse tema justifica-se diante da necessidade de conhecermos melhor o objeto direto da educação física, discutir mais sobre sua relevância e importância no âmbito da saúde e educação, uma vez que a educação física entra como forma de prevenção da obesidade, levando atividades físicas e práticas corporal.

Sabemos que a obesidade pode ter início em qualquer época da vida, principalmente nos períodos de aceleração do crescimento da criança quando ela começa a ingerir vários tipos de alimentos, por vezes inadequados. Daí a importância das aulas de Educação Física Escolar, pois estas aulas são o momento mais propício para o estabelecimento de vínculos fundamentais entre a prática regular de atividade física e a alimentação voltada na melhoria da saúde como um todo (OLIVEIRA, 2015).

As causas que explicam o fenômeno da obesidade infantil encontram respaldo nos fatos de que após o período de atividade escolar as crianças estão cada vez mais sedentárias e optam pelas as tecnologias e seus benefícios, que traz comodidade fazendo que cada vez mais jovens fiquem dentro de suas casas, jogando ou interagindo virtualmente, tornandoas assim, em crianças sem hábitos para a prática de exercícios físicos e consequentemente soma-se ainda a alimentação incorreta que dá preferência a alimentos hipercalóricos pouco nutritivos ou saudáveis.

Diante da necessidade de encontrar soluções para esse problema, este estudo objetivou analisar, por meio de uma revisão de literatura, a importância da educação física escolar e suas contribuições no controle da obesidade infantil visto que ela está se tornando um problema cada vez mais frequente na infância e na adolescência estando associada a inúmeras doenças graves. Assim, busca-se responder, com base na produção científica 
publicada em periódicos indexados, a seguinte pergunta norteadora da investigação: Como a Educação Física pode ajudar a combater a obesidade infantil e qual é o papel do professor de educação física no controle dessa doença?

\section{Metodologia}

O método empregado para a realização deste estudo foi uma revisão da literatura baseada em materiais encontrados nas seguintes bases de dados: SCIELO (Scientific Electronic Library Online), LILACS (Literatura Latino-americana e do Caribe em Ciências da Saúde) e bibliotecas digitais, entre outros. Foi feita a análise qualitativa e revisões sistemáticas em livros e artigos publicadas nos últimos 12 anos (2009-202I) que discutem sobre obesidade, saúde, importância da educação física escolar, além do papel coadjuvante do profissional de educação física relacionado aos objetivos propostos. Optou-se por estas bases de dados e biblioteca por entender que abrangem a temática abordada.

A busca ocorreu no período entre setembro e novembro de 2021, sendo usada como estratégia de busca os seguintes descritores: Obesidade, Educação Física, Qualidade de Vida, Hábitos Saudáveis e Atividade Física.

Outro critério também utilizado foi o ano de publicação dos estudos e artigos (2009-2021), artigos que apresentavam pesquisas originais, metanálises, livros digitais e publicações na Língua Portuguesa. Os estudos duplicados ou sem critérios de fontes de dados e textos não científicos, tais como: blogs, sites não indexados ou sem vínculo científico, foram descartados por não atender aos critérios de busca.

\section{Fundamentação teórica}

\section{I Obesidade}

\section{I.I Conceito e epidemiologia}

A obesidade, segundo as novas diretrizes literárias, é definida como sendo uma condição patológica, de etiologia multifatorial e de caráter crônico adquirida pelo ganho energético em excesso. Essa condição que acaba conferindo um acúmulo de tal ganho em forma de gordura em meio ao tecido adiposo, afetando diversos pontos de funcionamentos 
fisiológico dos sistemas corporais e, assim, desequilíbrio orgânico do indivíduo, deixando-o propenso ao desenvolvimento de diversas outras co-morbidades (GUYTON; HALL, 20II).

Os agravos provocados por essa condição patológica têm alavancado uma série de preocupações no cenário da saúde pública mundial, principalmente ao que tange as preocupações fomentadas pela organização mundial de saúde (OMS) quanto ao risco de mortalidade às mais variadas faixas etárias (SANTOS; RABINOVICH, 20II).

Considerada uma doença complexa a obesidade apresenta graves dimensões sociais e psicológicas, e pode afetar todas as faixas etárias e grupos socioeconômicos. No que lhe diz respeito, é reputada como uma doença não transmissível, que tem como características: longo período de latência, longo curso assintomático, curso clínico em geral lento, prolongado e permanentes manifestações clínicas com períodos de remissão e de exacerbação e de múltiplas determinações, com forte componente ambiental (LOPES et al., 2010).

A obesidade por sua vez tem causas multifatoriais e onde pode está associada a interação de fatores genéticos, metabólicos, sociais, comportamentais e culturais. São vários os casos que estão plenamente ligados ao abuso da ingestão calórica e ao sedentarismo, onde o excesso de calorias se armazena como tecido adiposo, gerando o balanço energético positivo. O balanço energético pode ser estipulado como a diferença entre a quantidade de energia adquirida e gasta pelo indivíduo na realização das funções vitais e de atividades em geral. Sendo positivo quando a quantidade de energia adquirida é maior do que a gasta, podendo variar entre pessoas (TAVARES; NUNES; SANTOS, 2010).

\subsubsection{Classificação e diagnóstico da obesidade}

A obesidade pode ser classificada, a partir de sua origem em: exógena e endógena. A obesidade do tipo endógena, está relacionada a problemas hormonais ou doenças endocrinas e não é a mais frequente; para sua intervenção deve-se identificar a doença de base e tratá-la, sendo responsável por 2,0\% dos casos. A obesidade exógena origina-se do desequilíbrio entre ingestão e gasto calórico, devendo ser manejada com orientação alimentar, especialmente mudanças de hábitos e otimização da atividade física. Esse tipo de obesidade é responsável por 98\% dos casos (CARVALHAL, 2013). 
A obesidade exógena na infância é um distúrbio nutricional de cunho multifatorial, influenciável por fenômenos como desmame precoce, a presença de sobrepeso na família, a alimentação excessiva, a baixa condição sócio econômica e distúrbios na dinâmica familiar (ESCRIVÃO et al., 2000 apud NERI et al., 2017).

Para diagnosticar a obesidade, vários métodos e técnicas podem ser utilizados. Esses estão divididos em dois grupos: diagnósticos quantitativos e diagnósticos qualitativos de obesidade.

Fung (2018), sintetiza cada um deles: (a) Métodos quantitativos: cálculo de IMC, tabelas do IMC, medição das pregas cutâneas, impedância bio elétrica de frequência única, espectroscopia bio elétrica de frequência múltipla, condutibilidade elétrica corpórea total, absorpciometria dual de raios $x$, tomografia computorizada e ressonância nuclear magnética, potássio corpóreo total (4ok), água duplamente marcada (d2o); (b) Métodos qualitativos: Medida do maior perímetro abdominal, relação cintura e quadril absorpciometria dual de raios $\mathrm{x}$ (dxa), ultrassonografia, tomografia computorizada $\mathrm{e}$ ressonância nuclear magnética.

A partir IMC, a obesidade pode ser dividida entre quatro grupos: (a) Obesidade tipo I, caracterizada pelo excesso de massa gorda total sem nenhuma concentração particular de gordura numa certa região corporal; (b) Obesidade tipo II, caracterizada pelo excesso de gordura subcutânea na região abdominal e do tronco (androide); (c) Obesidade tipo III, caracterizada pelo excesso de gordura víscero-abdominal; (d) Obesidade tipo IV, caracterizada pelo excesso de gordura glúteo-femural (ginóide) (IZIDORO; PARREIRA, 2010).

É importante considerar ainda que o excesso de gordura no organismo pode levar ao desenvolvimento de uma série de doenças secundárias, como o diabetes tipo 2, doenças cardiovasculares, hipertensão, artrite, apneia e derrame. Entretanto, nem todo caso de obesidade está associado a esses quadros de saúde.

A melhor estratégia para se controlar a obesidade é o diagnóstico precoce e a prevenção, com isso as pessoas que tem um convívio diário com as crianças, devem estar sempre vigilantes. Quanto antes identificar o sobrepeso, mais facilmente poderá eliminar seus fatores causadores. $O$ ideal seria que todas as áreas que tem contato direto com a 
criança, ou seja, a família, escolas, creches, pediatras, etc., estivessem atentas para a questão da obesidade, seus fatores de riscos e medidas de prevenção (CARVALHAL, 2013).

\subsection{Obesidade infantil}

A obesidade infantil acontece quando uma criança está com peso maior que o recomendado para sua idade e altura. A obesidade infantil está a atingir, nos dias de hoje, proporções cada vez mais preocupantes em todos os países do mundo, por isso a sua prevenção considera-se primordial. De acordo com a DGS (2017) a obesidade é considerada uma doença crónica e ao mesmo tempo um fator de risco para o desenvolvimento de outras doenças crónicas que constituem as principais causas de mortalidade e morbilidade.

A obesidade infantil é considerada a epidemia do século XXI pela OMS, representando atualmente um dos mais sérios problemas de saúde pública, que afeta tanto as crianças como os adolescentes, constituindo-se como um dos mais urgentes e sérios desafios para a saúde, pelo que a prevenção é a melhor forma de combater esta realidade (OMS, 2016).

São diversas as complicações associadas à obesidade infantil, não só pelas implicações que o excesso de peso tem para a estrutura corporal, mas também pelas perturbações a nível metabólico causadas pela mesma. As complicações associadas à obesidade infantil geralmente só se manifestam na idade adulta, existindo um risco considerável de morbilidade e mortalidade, verificando-se um aumento do número de crianças e adolescentes que apresentam graves complicações de saúde (PINTO, 2017).

A obesidade infantil afeta o normal crescimento e desenvolvimento da criança e acarreta inúmeras complicações futuras, que se podem dividir em complicações físicas (doenças cardiovasculares, diabetes, hipertensão, em alterações gastrointestinais, ortopédicas, respiratórias e dermatológicas) e psicológicas (discriminação, depressão e dificuldades na interação social) (EBBELING; PAWLAK; LUDWIG; 2002 apud JANEIRO, 2020).

Assim, as crianças ficam mais vulneráveis, pois tanto as complicações físicas como as psicológicas, afetam de forma negativa a criança, contribuindo para diminuição da sua autoestima (SALIM; BICALHO, 2004 apud JANEIRO, 2020). 


\subsubsection{Causas e consequências da obesidade infantil}

Pode-se afirmar que a obesidade infantil depende de dois fatores. O primeiro é o fator genético (endógeno), formado pelo conjunto de genes herdados através dos pais. Mesmo que os genitores não sejam obesos, sua prole pode carregar esta carga genética. Quanto menos idade tiver e mais pesada for a criança, terá uma maior probabilidade de ter estas especificidades genéticas. $O$ segundo aspecto é a influência do meio ambiente (fator exógeno). As crianças que ficam muito em casa, atentas a TV e videogames, vivem na verdade uma vida sedentária, o que contribui muito para a obesidade infantil (OLIVEIRA; COSTA, 2016).

Além do mais, o estilo de vida e os fatores nutricionais, como escolha dos alimentos, bem como quantidades e frequência que são ingeridos, são os aspectos mais determinantes para o quadro da obesidade infantil. Conforme diversos estudos na área, atualmente são evidentes o baixo consumo de verduras, legumes e frutas e o aumento no consumo de açúcar refinado e refrigerante. A substituição de refeições balanceadas por lanches rápidos, com valores nutricionais inadequados agrava muito também este quadro (SOARES et al., 20II).

A causa primária da obesidade é o desequilibro crônico entre a ingestão alimentar e o gasto energético, o que resulta de elevado consumo calórico e pouca atividade física, o que nos leva a acreditar que a combinação de dieta e exercícios pode proporcionar perda de peso mais eficiente durante curto ou longo prazo, em comparação à apenas uma dessas intervenções isoladamente. Mas não podemos esquecer que a obesidade infantil está diretamente associada ao sedentarismo (MEIRELLES; GOMES, 2004 apud LIMA, 2013).

É extremamente necessário identificar as causas da obesidade de cada indivíduo, entretanto descobrir o efeito desencadeador da obesidade se tornou algo muito difícil, entretanto, os fatores internos e externos estão entre os fatores que mais a influencia (LIMA, 2013).

Entre esses fatores, podemos citar: consumo de produtos ricos em gorduras com alto valor calórico, diminuição da prática de exercícios físicos, tempo de televisão diária e avanços tecnológicos da sociedade moderna. A prática de assistir à televisão durante várias horas por dia, os jogos eletrônicos, o abandono da amamentação são fatores que devem ser 
considerados na determinação do crescimento da obesidade infantil (TADDEI, 1995 apud HERNANDES; VALENTINI, 2010).

As principais consequências para a criança obesa são: a elevação dos triglicérides e do colesterol, alterações ortopédicas (joelhos valgos), pressóricas, dermatológicas e respiratórias, sendo que, na maioria das vezes, essas alterações se tornam mais evidentes na vida adulta. Pesquisas apontam que cerca de 50\% das crianças obesas apresentam alterações na taxa de colesterol; 47,5\% têm níveis diminuídos de HDL e 20,5\% têm níveis elevados de LDL (PIRES et al., 2015).

O aumento da adiposidade corporal em crianças, além de causar sérios riscos à saúde, pode prejudicar a sociabilidade e o estado emocional, pois estas passam a enfrentar grande pressão negativa desde idades precoces. As interferências da obesidade sobre a personalidade da criança são inúmeras (COSTA et al., 2012).

Quanto mais tempo os indivíduos se mantém obesos, maior é a chance de complicações futuras ocorrerem. Crianças em idade pré-escolar que já apresentam quadro de obesidade, têm cinco vezes mais chance de serem adolescentes obesos e quatro vezes

mais chance de serem adultos obesos quando comparadas a crianças que se encontram com o peso adequado (JANEIRO, 2020).

De acordo com Carvalhal (2013), a criança obesa geralmente não é feliz, pois se sente frágil, desamparada e solitária, sofrendo com as brincadeiras e apelidos colocados pelos colegas, tornando-se insegura e arredia ao contato (especialmente com os mais próximos). Transtornos psicossomáticos podem acompanhar a doença como: diarreias, bronquite, cefaleias, vertigem e constipação intestinal. Por isso, a prevenção da obesidade infantil é muito importante. E isso se previne com uma alimentação saudável e a prática de atividades físicas.

\subsubsection{Tratamento da obesidade infantil}

O tratamento da obesidade tem por objetivo alcançar a redução de peso, mas não o peso ideal e sim um peso saudável, no qual as complicações associadas à obesidade são nulas ou mínimas. Para isso, é necessário adoção de mudança de hábitos de vida como reeducação alimentar, prática de atividades físicas. Em alguns casos, avaliados por 
profissionais, há a necessidade de intervenção medicamentosa ou cirúrgica (CARVALHAL, 2013).

Para Silva (20II), o tratamento da obesidade em crianças é bem mais complicado que a dos adultos, pois envolve vários aspectos e é sobretudo comportamental, enfocando reeducação nutricional, mudanças no estilo de vida e aumento da prática de exercícios físicos. Durante a reeducação alimentar devem ser disponibilizadas dietas flexíveis, que atendam às necessidades alimentares da criança, visto que dietas muito rígidas se mostram ineficientes, ocasionando prejuízos ao desenvolvimento, além de maiores índices de insucesso. A atividade física deve ser praticada de três a cinco vezes por semana, com duração de 50 a 60 minutos. Os exercícios físicos preservam a massa magra e minimizam a redução da taxa metabólica. Um ponto importante é que toda a família deve estar envolvida e não pode ter intervenções farmacológicas agressivas.

De modo geral, o tratamento da obesidade infantil, deve ser eficiente, não somente para estética e diminuição da massa corporal e sim voltado para relações psicopatológicas. A terapia cognitivo-comportamental é muito usada no tratamento da obesidade infantil. É um método de duração breve, no qual o paciente é solicitado a colaborar nas mudanças de seus próprios atos que contribuem para sua obesidade. $O$ tratamento utiliza registros alimentares, técnicas de modificação de hábitos alimentares, estratégias para minimizar o contato com situações de consumo inadequado da comida, treinamento para solução de problemas, além da prevenção de recaídas. Através destas atitudes, a criança melhora sua integração com o ambiente familiar e social, modificando seus hábitos para que sejam os mais saudáveis possíveis (PARRA; MOTA, 2012).

\subsection{Educação física escolar e obesidade infantil}

Os alarmantes índices de obesidade infantil registrados no Brasil demonstram como é importante a prevenção do sobrepeso, sendo a escola o local considerado ideal para realizar as intervenções necessárias, principalmente através da Educação Física. Como disciplina que se caracteriza por promover a saúde, os profissionais devem atuar como impulsionadores de seus alunos, para que sempre adotem um estilo de vida ativo. A prática regular de exercícios físicos durante a fase escolar incentiva a adoção de hábitos saudáveis durante toda vida (ARAÚJO; BRITO; SILVA, 20I0). 
De acordo com Lima (2013) a Educação Física, hoje em dia, não está mais limitada a conhecimento tático de alguns esportes. Atualmente, o objetivo da Educação Física é fazer as crianças e adolescentes enxergarem as mudanças de hábitos que influenciam o ganho de peso do mundo de hoje e não somente a cultura corporal.

Conhecer o quanto a prática de atividades físicas é importante para a saúde é extremamente necessário, visto que muitas crianças optam por assistir televisão e brincar com jogos eletrônicos do que correr, pular, jogar bola. A inatividade física durante a infância é muito prejudicial. Sendo assim, a Educação Física, deve assumir o papel de auxiliar na saúde das crianças, uma vez que, o objetivo da aula é desenvolver habilidades e capacidades físicas. É de extrema importância esclarecer as crianças sobre os bons hábitos alimentares e a prática de atividades e exercícios físicos durante sua vida como forma de prevenção da obesidade (GUIDO; MORAES, 2010).

Segundo Silva (2011) a atividade física ocasiona grandes benefícios a saúde de quem a pratica, como a redução do peso e gordura corporal, aumento da taxa metabólica basal, utilização das reservas lipídicas, melhora da capacidade aeróbica, regulação do apetite, melhor imagem e expressão corporal e também uma maior integração social, além de trazer benefícios psicológicos, entre eles: autoconceito, autoconfiança, autoestima, diminuição da ansiedade e também de níveis de depressão.

Evidências apontam que atividade física quando bem estruturada com objetivos bem definidos podem alcançar benefícios satisfatórios a saúdes de crianças e adolescentes em idade escolar, sendo que qualquer atividade moderada já seria capaz de promover ganhos satisfatórios à saúde em crianças em idade escolar, induzindo assim a melhor qualidade de vida com mais disposição para realização das tarefas diárias, redução de fatores de riscos à saúde como obesidade, doenças coronarianas, diabetes, além de câncer, depressão, e problemas articulares (RIBAS FILHO; ALMEIDA, 202I).

\subsection{O professor de educação física}

Conforme os Parâmetros Curriculares Nacionais cabe à Educação Física escolar a responsabilidade de lidar de forma específica com alguns aspectos relativos aos conhecimentos procedimentais, conceituais e atitudinais característicos da cultura corporal de movimento, bem como identificar os alunos com sobrepeso ou com tendência a 
obesidade, é que o profissional de educação física pode desenvolver atividades que contemplem todos os alunos, infelizmente há uma grande maioria que é responsável em identificar essa situação e não o fazem (BRASIL, I998 apud OLIVEIRA; COSTA, 2016).

Nesse sentido, o papel da Educação Física ultrapassa o ensinar esporte, ginástica, dança, jogos, atividades rítmicas, expressivas e conhecimento sobre o próprio corpo para todos, mas inclui também os seus valores subjacentes, ou seja, quais atitudes os alunos devem ter nas e para as atividades corporais. E, finalmente, busca garantir o direito do aluno de saber o porquê dele realizar este ou aquele movimento, isto é, quais conceitos estão ligados àqueles procedimentos voltados à consciência de uma vida saudável (CARVALHAL, 2013).

Atualmente, o professor/educador físico, é considerado um profissional da saúde tem condições de acatar estratégias que trabalhe na identificação, prevenção e tratamento de enfermidades, dentre elas a obesidade infantil, o mesmo deve dispor de uma atenção especial e ter ciência de responsabilidade, na medida em que já é comprovado que o sedentarismo está entre as principais causas do aumento dos índices de obesos no Brasil (OLIVEIRA; COSTA, 20I6).

Quando os alunos entendem a importância da luta ao sedentarismo e que esse é uma das causas mais relevantes da obesidade, os professores conseguem atingir esses alunos na prática de atividade física, gerando a conscientização da saúde e consequentemente minimizando expressões negativas de sentimentos (LIMA, 2013).

Com a interação da teoria e prática da atividade física sobre a obesidade infantil, o professor de educação física desenvolve um trabalho de conhecimento, vivência e conscientização desse problema que atinge crianças e adolescentes no Brasil e no mundo. Sendo assim, diferenciar informações de conhecimento é a verdadeira forma de conscientizar e através da aprendizagem eficaz é que a criança se transforma num agente multiplicador (SILVA; BEZERRA, 2017).

O trabalho de orientação e prevenção, pode ser feito pelo professor de educação física, trabalhando de forma interdisciplinar com outros professores, Mello, et al. (2004 apud CARVALHAL, 2013) diz que prevenir a obesidade infantil significa diminuir, de forma racional e barata, a incidência de doenças crônico-degenerativas no futuro. Nesse sentido a aula de educação física atuam como forma de conscientização dos alunos e 
familiares, uma vez que a conscientização e a prática da atividade física traz vários benefícios a vida do aluno.

O professor de Educação Física deve sempre orientar os pais a serem modelos de um estilo de vida saudável para seus filhos e reduzir o tempo de "tela": televisão, videogames, computador e celulares a menos de duas horas ao dia nos períodos em que a criança ou o adolescente não está na escola e tem por dever acompanhar o desenvolvimento dos escolares através de seus métodos de avaliação física, buscando encorajar os alunos a realizarem os exercícios físicos e compromisso com hábitos alimentares saudáveis, buscando beneficiar sua saúde (BRAVIN et al., 2016).

Professores e demais profissionais envolvidos com a educação devem ser encorajados a estabelecer políticas internas de promoção de atividades físicas agradáveis e adaptadas a cada faixa etária, desde o ensino fundamental até o ensino médio, bem como criar programas de educação em saúde para crianças e adolescentes. Além disso, deve obter recursos adequados (incluindo pessoal), equipamentos e adequação dos espaços físicos; contratar profissionais preparados e treinados tanto para as aulas de educação física e atividades físicas, bem como professores capacitados em educação em saúde, promovendo um ambiente físico e social favorável, atrativo e encorajador a atividades físicas (GUIDO; MORAES, 2010).

Prevenir a obesidade é primordial para a saúde do escolar. É fundamental que a educação física esteja integrada ao currículo formal da escola, ensinando aos estudantes sobre os hábitos de vida saudáveis, estimulando as transformações em relação aos costumes nocivos, buscando as práticas de hábitos benéficos (CORNACHIONI; ZADRA; VALENTI, 20II).

Para os autores Guido e Moraes (2010), a prevenção deve estar vinculada ao sistema educacional, juntamente com a colaboração da família, com o entendimento de que, a prevenção e o tratamento eficazes, se dão por meio da dieta e do exercício físico. Para isto, é importante que o profissional de Educação Física e o grupo familiar possam compartilhar diversas informações sobre a temática, buscando entusiasmar a criança quanto à importância do consumo de alimentos saudáveis e instigar para a prática de exercícios físicos. 
Criar programas extracurriculares de atividades físicas diversificadas, incluir a participação dos pais nos programas extracurriculares e prover educação continuada para profissionais (professores, treinadores, recreacionistas e administradores), sobre a importância de estimular a prática de atividades físicas e incorporar essa rotina ao longo da vida dos jovens é papel da escola (BENEDITO, et al., 2014).

Segundo Balbinotti et al. (20II) por meio da prática pedagógica da educação física os alunos deverão ter consciência da importância da atividade física regular, sendo que, as aulas deverão apresentar diversas modelos de atividades a serem desenvolvidas, promovendo a saúde. Além disso, estas atividades devem ser variadas e motivar o jovem para adquirir consciência corporal, objetivando a queima de gordura corporal, evitando assim exercícios repetitivos, estimulando diferentes níveis de dificuldades.

\section{CONSIDERAÇÕES FINAIS}

É consenso que a obesidade infantil vem aumentando de forma significativa e que ela determina várias complicações na infância e na idade adulta. Na infância, o manejo pode ser ainda mais difícil do que na fase adulta, pois está relacionado a mudanças de hábitos e disponibilidade dos pais, além de uma falta de entendimento da criança quanto aos danos da obesidade.

Uma das principais causas da obesidade infantil é o sedentarismo, juntamente com a alimentação inadequada. Nesse sentido, a prevenção, os exercícios físicos estimulados desde a infância e uma dieta (ambos combinados) são mais eficazes no combate à obesidade infantil, promovendo saúde e bem estar.

Conclui-se que a Educação Física, voltada para uma proposta de educação que influencie na prevenção por meio da informação e do incentivo, a fim de buscar estilos de vida saudáveis, se apresenta como meio bastante eficaz na ação de combate a obesidade infantil, uma vez que um dos maiores causadores do excesso de peso é a inatividade física. Assim, fica claro que a educação física escolar é responsável por educar nossas crianças e adolescentes em toda a sua trajetória no ensino básico, sobre como tratar e/ou cuidar de seu corpo numa dimensão física, trazendo-lhes o conhecimento sobre os benefícios preventivos da atividade física em relação ao corpo saudável. 


\section{REFERÊNCIAS}

ARAÚJO, R. A.; BRITO, A. A.; SILVA, F. M. O papel da educação Física escolar diante da epidemia da obesidade em crianças e adolescentes. Educação Física em Revista, v. 4, n.2, maio/ago. 2010.

ATAlLA, M. Os fatores da obesidade Infantil. BemStar com Marcio Atalla, 9'38”, 2016. Disponível em: 〈https://www.youtube.com/watch?v=v5Ilik-KRSA〉. Acesso em $25 / 10 / 2021$.

BALBINOTTI, M. A. A.; et al. Motivação à prática regular de atividades físicas e esportivas: um estudo comparativo entre estudantes com sobrepeso, obesos e eutróficos. Motriz, Rio Claro, v.17, n. 3, p. 384-394, jul./set., 201 .

BENEDITO, L. S.; et al. Educação física escolar: no combate à obesidade infantil. Revista Científica Multidisciplinar Núcleo Do Conhecimento, v. Io, Ano I, 2014.

BRASIL, Ministério da Saúde. Secretaria de Atenção à Saúde. Departamento de Atenção Básica. Política nacional de alimentação de nutrição. Brasília: Ministério da Saúde, (Série B. Textos Básicos de Saúde), 2012. 48 p.

BRAVIN, M. B.; ROSA, A. R.; PARREIRA, M. B.; PRADO, A. F. A influência do exercício físico na obesidade infantil. Revista Ciência e Estudos Acadêmicos de Medicina, [S. l. $], \quad$ v. $\quad$ I, $\quad$ n. 04, 2016. Disponível em: $\langle$ https://periodicos.unemat.br/index.php/revistamedicina/article/view/847〉. Acesso em: $5 / \mathrm{II} / 202 \mathrm{I}$.

CARVALHAL, M. I. M. Obesidade infantil e atividade física. I. ed., Curitiba (PR): CRV, 2013. $188 \mathrm{p}$.

CORNACHIONI, T. M.; ZADRA, J. C. M.; VALENTIM, A. A obesidade infantil na escola e a importância do exercício físico. Lecturas Educación Física y eportes, Buenos Aires, Ano i6, n.I57, jun. 20Ir.

COSTA, M. A. P.; SOUZA, M. A.; OLIVEIRA, V. M. Obesidade infantil e bullying: a ótica dos professores. Educação e Pesquisa, São Paulo, v. 38, n. 3, p. 653-665, 2012.

DGS. Direção-Geral da Saúde / Programa Nacional para a Promoção da Alimentação Saudável. Obesidade: otimização da abordagem terapêutica no serviço nacional de saúde. Lisboa, Portugal: DGS. 2017. $20 \mathrm{p}$. 
FUNG, J. O código da obesidade: decifrando os segredos da prevenção e cura da obesidade. I. Ed., São Paulo: nVersos, 2018. 256 p.

GUIDO, M; MORAES, J. F. Educação Física Escolar: Como Prevenção e Tratamento para o Sobrepeso e Obesidade infantil: uma revisão. Revista Digital, Buenos Aires, ano I5, n. 146, jul. 2010.

GUYTON, A. C; HALL, J. E. Tratado de fisiologia médica. I2. ed. Rio de Janeiro: Elsevier, 20II. II20 p.

HERNANDES, F.; VALENTINI, M. P. Obesidade: causas e consequências em crianças e adolescentes. Conexões, Campinas, SP, v. 8, n. 3, p. 47-63, 2010.

IZIDORO, F. G.; PARREIRA, N. S. Obesidade infantil. 2010. 23 p. Trabalho de Conclusão de Curso (Graduação em Técnico de Enfermagem), Instituto Federal de Educação, Ciência e Tecnologia, Capetinga, zoı.

JANEIRO, A. I. C. Obesidade infantil: a atuação do enfermeiro especialista em enfermagem de saúde infantil e pediátrica na educação alimentar em idade escolar. 2020. i64 p. Relatório de Estágio (Mestrado em Enfermagem de Saúde Infantil e Pediatria),

Escola Superior de Enfermagem São João de Deus da Universidade de Évora, Portalegre, 2020 .

LIMA, J. G. O. A importância da educação física escolar na prevenção da obesidade em crianças e adolescentes. 2013. Io p. TCC (Graduação em Educação Física), Faculdade de Ciências e Saúde, Centro Universitário de Brasília, 2013.

LOPES, P.C.S. et al. Fatores de risco associados à obesidade e sobrepeso em crianças em idade escolar. Rev Bras Enferm, Brasília, v. 63, n. I, 6 p., jan-fev, 2010.

MALTA, D. C. Panorama atual das doenças crônicas no Brasil. Brasília: Secretaria de Vigilância em Saúde/Ministério da Saúde; 20II. 50 p.

NASCIMENTO, M. B.et al. Adolescent obesity: a profile currently outlined through narrative review. Research, Society and Development, [S. l.], v. io, n. I, p. e26710iri857, 2021. DOI: I0.33448/rsd-vioir.II857. Disponível em: 〈https://rsdjournal.org/index.php/rsd/article/view/II857〉. Acesso em: 28/II/202I.

NERI, L. C. L. et al. Obesidade infantil. I. ed., São Paulo: Manole, 2017. 152 p.

OLIVEIRA, D. M. Disfunções Orgânicas da Atividade Física e Saúde. Centro Universitário Claretiano, Batatais, São Paulo, Unidade I, 2015 . 122 p. 
OLIVEIRA, L. F. L.; COSTA, C. R. B. Educação física escolar e a obesidade infantil. Revista Científica Multidisciplinar Núcleo Do Conhecimento, v. ıo, Ano I, p. 87-1o I, Nov. 2016.

OMS, Organização Mundial de Saúde. Relatório mundial da saúde. Financiamento dos sistemas de saúde - o caminho para a cobertura universal. 2010. I43 p.

OMS, Organização Mundial de Saúde. Report of commission on ending childhood obesity. Genebra, Suíça: OMS, 2016. 68 p.

PARRA, E. M.; MOTA, G. R. Obesidade infantil: causas, consequências e a prática de atividade física na prevenção e tratamento. Efdeportes.com, ano 16, n. 165, fev. 2012.

PINTO, L. Conhecimento dos Pais sobre alimentação infantil: relação com as caraterísticas sócio demográficas e estado nutricional da criança. 2017. I43 p. Dissertação de Mestrado (Mestrado em Enfermagem de Saúde Infantil e Pediatria), Escola Superior de Saúde de Viseu do Instituto Politécnico de Viseu, Viseu, Portugal, 2017.

PIRES, A.; MARTINS, P.; et al. Células progenitoras endoteliais em crianças e adolescentes obesos. J Pediatra (Rio J), v. 91, p. 560-612, 2015.

RIBAS FILHO, D.; ALMEIDA, C. A. N. Livro - texto de obesidade: uma visão clínica e abrangente da ABRAN. I. ed., São Paulo: Manole, 2021. 56o p.

SANTOS, L. R. C.; RABINOVICH, E. P. Situações familiares na obesidade exógena infantil do filho único. Saúde e Sociedade, v. 20, n. 2, p. 507-521, 2011.

SBP, Sociedade Brasileira de Pediatria. Departamento de nutrologia, obesidade na infância e adolescência: manual de orientação. Departamento Científico de Nutrologia. 3. ed., São Paulo: SBP. 2019. 240 p.

SERRA, I. B. M. Obesidade infantil em escolares. 2017. 26 p. Trabalho de Conclusão de Curso (Graduação em Licenciatura em Educação Física), Faculdade de Ciências da Educação e Saúde Centro Universitário de Brasília - UniCEUB, Brasília, 2017.

SILVA, D. A. S. Sobrepeso e obesidade em crianças de cinco a dez anos de idade beneficiárias do Programa Bolsa Família no estado de Sergipe, Brasil. Revista Paulista Pediatria, São Paulo, v. 29, n. 4, dez. 201 .

SILVA, F. A. L.; BEZERRA, J. A. X. Benefícios da atividade física no controle da obesidade infantil. Revista Campo do Saber, v. 3, n. I, p. 20I-218, jan/jun, 2017. 
SOARES, G. C et al. Os fatores que influenciam na obesidade infantil: uma revisão de literatura. 2011. Disponível em: $<$ http://189.59.9.179/cbcenf/sistemainscricoes/arquivosTrabalhos/I40163.Eıo.T7059.D6AP. pdf $>$. Acesso em I5/10/2021.

TAVARES, T. B, NUNES, S. M; SANTOS, M. O. Obesidade e qualidade de vida: revisão da literatura. Rev Med, Minas Gerais, v. 20, n. 3, p. 359-366, 2010. 